\title{
Recovery of Adrenal Function after Pituitary Surgery in Patients with Cushing Disease: Persistent Remission or Recurrence?
}

\author{
Andreea Liliana Serban ${ }^{a, b}$ Elisa Sala a, c Giulia Carosia, c Giulia Del Sindaco a, c \\ Claudia Giavolia Marco Locatellid Maura Arosio ${ }^{a, c}$ Giovanna Mantovania, c \\ Emanuele Ferrante ${ }^{a}$ \\ ${ }^{a}$ Endocrinology Unit, Ospedale Maggiore Policlinico, Fondazione IRCCS Ca' Granda, Milan, Italy; ${ }^{\text {b Department of }}$ \\ Experimental Medicine, Sapienza University of Rome, Rome, Italy; ${ }^{C}$ Department of Clinical Sciences and Community \\ Health, University of Milan, Milan, Italy; ${ }^{\mathrm{d}}$ Neurosurgery Division, Ospedale Maggiore Policlinico, Fondazione IRCCS \\ Ca' Granda, Milan, Italy
}

\author{
Keywords \\ Cushing disease $\cdot$ Adrenal insufficiency $\cdot$ Recurrence
}

\begin{abstract}
Background: Cushing disease (CD) represents the principal cause of endogenous hypercortisolism. The first-line therapy of $C D$ is surgical removal of the ACTH-secreting pituitary adenoma, which is generally followed by adrenal insufficiency (AI). Objective: To analyze the recovery of $\mathrm{Al}$ in patients with $C D$ after pituitary surgery in relation with recurrence and persistent remission of CD. Patients and Methods: We performed a retrospective analysis of patients with CD who met the following inclusion criteria: adult age, presence of $\mathrm{Al}$ 2 months after the surgical intervention, and a minimum follow-up of 3 years after the surgical intervention. Results: Sixty-one patients were followed for a median of 6 years. Ten (16.4\%) patients recurred during follow-up. The patients who restored adrenal function did so after a median time of 19 months, with a significantly shorter time in the recurrence
\end{abstract}

\section{KARGER}

(c) 2019 S. Karger AG, Basel

E-Mail karger@karger.com

www.karger.com/nen group (12.5 vs. 25 months, $p=0.008$ ). All 10 patients who recurred recovered their adrenal function within 22 months. The recovery rate of $\mathrm{Al}$ in the persistent remission group was $37.3 \%(19 / 51)$ at 3 years and $55.8 \%(24 / 43)$ at 5 years. In all patients the duration of Al was negatively associated with disease recurrence. Conclusion: The duration of postsurgical $\mathrm{Al}$ in patients with recurrent $\mathrm{CD}$ is significantly shorter than that in patients with persistently remitted $C D$, and this parameter may be a useful predictor of recurrence. Patients showing a normal pituitary-adrenal axis within 2 years after surgery should be strictly monitored as they are at higher risk of disease relapse.

(c) 2019 S. Karger AG, Basel

\section{Introduction}

Cushing disease (CD) represents the principal cause of endogenous hypercortisolism, a condition associated with important mortality and morbidity. In the absence

Andreea Liliana Serban

Endocrinology Unit, Ospedale Maggiore Policlinico, Fondazione IRCCS Ca' Granda Via Francesco Sforza, 35

IT-20143 Milan (Italy)

E-Mail andreea.l.serban@gmail.com 
of an adequate treatment, $50 \%$ of cases are fatal at an average of 4.5 years [1]. Furthermore, in a recent multicenter retrospective study, patients who achieved cure still showed an increased overall mortality risk, related in particular to cardiovascular and cerebrovascular diseases [2]. The first-line therapy of CD is the surgical removal of the ACTH-secreting pituitary adenoma. As recently reported, the median success rate of neurosurgery is $78 \%$ [3], arriving up to $96 \%$ in specialized centers [4]. Nevertheless, approximately one-third of cured patients experience recurrence of the disease during their lifetime [5], a condition that requires other therapeutic interventions such as reintervention, radiotherapy, medical therapy, or bilateral adrenalectomy. While patients cured by pituitary surgery alone seem to have a long-term survival similar to that of the general population, the increased number of treatments has a negative impact on survival even when remission is achieved [2].

A successful surgical intervention is generally followed by adrenal insufficiency (AI) due to the suppressive effect of cortisol on the hypothalamic-pituitary-adrenal (HPA) axis or alternatively to pituitary injury occurring during neurosurgery. Hypopituitarism, including secondary AI, represents another factor that could increase the mortality of patients cured from hypercortisolism [6]. Hypoadrenalism may last from months to years and in some cases is permanent. In this context, normalization of basal and stimulated plasmatic cortisol that occurs during follow-up of surgically cured patients may represent the recovery of the HPA axis or the onset of CD recurrence. It was demonstrated that patients who recurred during follow-up recovered their adrenal function earlier than patients with persistent remission [7], but previous studies assessing the duration of AI after successful pituitary surgery always included the relapsed cases [7-11]. This may lead to an underestimation of the overall duration of AI.

The aim of this study was to analyze the recovery of adrenal function in patients with CD after pituitary surgery in relation with recurrence and persistent remission of CD.

\section{Patients and Methods}

We performed a retrospective analysis of patients with $\mathrm{CD}$ followed in our center (Endocrinology Unit, Ospedale Maggiore Policlinico, Fondazione IRCCS Ca' Granda) between 1990 and 2017 who met the following inclusion criteria: age at surgical intervention $>18$ years, presence of AI 2 months after the surgical intervention, and a minimum follow-up of 3 years after the surgical intervention.
The diagnosis of $\mathrm{CD}$ was made on the basis of the presence of typical signs and symptoms of hypercortisolism, along with biochemical evaluation including ACTH and urinary free cortisol (UFC) levels, low- and high-dose dexamethasone (DXM) suppression tests, corticotropin-releasing hormone stimulation test, and bilateral inferior petrosal sinus sampling with corticotropin-releasing hormone when appropriate. Histopathological diagnosis of a corticotroph tumor and clinical and biochemical remission following transsphenoidal surgery (TSS) confirmed the diagnosis. Magnetic resonance imaging was done in all patients.

All neurosurgical interventions were executed using the TSS approach combined with the microscopic method until 2008 and with the endoscopic method after 2008. The modification of the surgical technique in our center was previous analyzed and the authors concluded that the two techniques were similar in terms of surgical safety [12]. In addition, in a recent meta-analysis, endoscopic surgery did not show a significantly higher rate in terms of gross total tumor resection in comparison to microscopic surgery for functioning pituitary adenomas [13]. The goal of the neurosurgical team for the first TSS was adenomectomy, hemi-hypophysectomy or total hypophysectomy being reserved for cases that required surgical reintervention. Immediately after TSS, all patients received a standard therapy with $25 \mathrm{mg}$ cortisone acetate per day divided in two or three doses. Patients were evaluated 2 months after pituitary surgery, then every 6 months in the first 3 years and once a year thereafter.

The diagnosis of AI was based on morning serum cortisol levels $<3 \mu \mathrm{g} / \mathrm{dL}$ or lack of cortisol response to a $1 \mu \mathrm{g} / 250 \mu \mathrm{g}$ Synacthen stimulation test, using a cutoff value of $18 \mu \mathrm{g} / \mathrm{dL}$. Until 1998, adrenal function was studied with a $250 \mu \mathrm{g}$ Synacthen test. After 1998, after the demonstration at our institution of its usefulness [14], a $1 \mu \mathrm{g}$ Synacthen test was adopted as the routine test in patients with pituitary diseases.

The baseline cortisol and the Synacthen stimulation test were performed at 8.00-8.30 a.m. in the fasting condition. Patients were advised to discontinue their substitutive therapy with cortisone acetate $24 \mathrm{~h}$ before the hormonal evaluation. In case of negligence, the evaluation was postponed to the next day. Other pharmacologic interferences such as the use of contraceptive pills, opiates, or other glucocorticoid treatments were excluded. Until 2009, serum cortisol levels were determined by an immunofluorometric assay (AutoDelfia kit; Wallac Oy, Turku, Finland). After 2009, an electrochemiluminescence immunoassay (Cobas; Roche Diagnostic GmbH) was used. The normal range and cutoff for the Synacthen tests were similar for both methods. After 2017, cortisol levels at our institution were measured with Roche Cortisol II. This method generates cortisol levels approximately 30\% lower than the older immunoassay, with a consequent change in cutoff levels to define hypoadrenalism. However, the patients studied in this case series were evaluated previously to the change of cortisol assay. All patients with AI were considered cured. The patients who recovered their adrenal function were screened immediately for recurrence, then every 6 months in the first 3 years and once a year thereafter.

Disease recurrence was defined as elevated UFC levels in at least two samples and a positive DXM suppression test (overnight $1 \mathrm{mg}$ or standard 2-day DXM suppression test, cortisol $>1.8 \mu \mathrm{g} / \mathrm{dL}$ ) associated with reappearance of clinical signs of disease. Because of high variability of the UFC normality range, we preferred to use relative UFC (UFC, upper normal limit ratio). 
Table 1. General characteristics of all patients divided in two subgroups according to CD recurrence

\begin{tabular}{|c|c|c|c|c|}
\hline Number of patients & 61 & 10 & 51 & - \\
\hline Mean age at diagnosis, years & $41 \pm 14$ & $42 \pm 14.6$ & $40.8 \pm 14$ & 0.74 \\
\hline Mean body mass index, $\mathrm{kg} / \mathrm{m}^{2}$ & $27.5 \pm 6.1$ & $25 \pm 3.6$ & $27 \pm 6.4$ & 0.2 \\
\hline \multicolumn{5}{|l|}{ Cortisol hypersecretion characteristics } \\
\hline Median relative UFC & $2.1(1.3-4.3)$ & $3.2(1.8-6.2)$ & $2.1(1.3-4.3)$ & 0.38 \\
\hline Mean cortisol after $1 \mathrm{mg} D X M, \mu g / d L$ & $13.8 \pm 8.1$ & $14 \pm 8.7$ & $13.7 \pm 8.1$ & 0.9 \\
\hline Median disease duration prior to $\mathrm{TSS}^{\mathrm{a}}$, months & $36(15-72)$ & $26(15.5-43.5)$ & $36(15-48)$ & 0.86 \\
\hline \multicolumn{5}{|l|}{ Radiologic adenoma features } \\
\hline Microadenoma & $43 / 61$ & $6 / 10$ & $37 / 51$ & 0.4 \\
\hline \multicolumn{5}{|l|}{ Immunohistochemical features } \\
\hline ACTH+ & $37 / 40$ & $1 / 6$ & $2 / 34$ & 0.4 \\
\hline \multicolumn{5}{|l|}{ Post-TSS characteristics } \\
\hline Mean baseline cortisol, $\mu \mathrm{g} / \mathrm{dL}$ & $2.7 \pm 2.1$ & $3.3 \pm 1.9$ & $2.6 \pm 2.1$ & 0.4 \\
\hline Cortisol after Synacthen $1 \mu \mathrm{g}, \mu \mathrm{g} / \mathrm{dL}$ & $8.6 \pm 3.7$ & $9.7 \pm 4.5$ & $8.2 \pm 3.6$ & 0.65 \\
\hline Cortisol after Synacthen $250 \mu \mathrm{g}, \mu \mathrm{g} / \mathrm{dL}$ & $7.7 \pm 4.8$ & & $7.7 \pm 4.8$ & \\
\hline Median follow-up, years & $6(4-11)$ & $7.5(3.75-11)$ & $6(4-11)$ & 0.97 \\
\hline Median recovery time from $\mathrm{AI}$, months & $19(6-31)$ & $12.5(7.8-18.2)$ & $25(12-40.9)$ & 0.008 \\
\hline Growth hormone insufficiency & $18 / 61$ & $1 / 10$ & $17 / 51$ & 0.25 \\
\hline Thyroid-stimulating hormone insufficiency & $13 / 61$ & $1 / 10$ & $12 / 51$ & 0.67 \\
\hline GN insufficiency & $13 / 61$ & $1 / 10$ & $12 / 51$ & 0.67 \\
\hline
\end{tabular}

Values in parentheses represent interquartile ranges. AI, adrenal insufficiency; CD, Cushing disease; DXM, dexamethasone; TSS, transsphenoidal surgery; UFC, urinary free cortisol. ${ }^{a}$ Disease duration was defined as the interval between the first signs and symptoms reported by the patient and the surgical intervention.

As mentioned above, a comprehensive study of pituitary function after TSS was assessed. Central hypothyroidism was diagnosed in the presence of low free thyroxine level associated with low or normal thyroid-stimulating hormone values. Central hypogonadism in men was defined by low total testosterone levels associated with normal or low gonadotropins (luteinizing hormone, follicle-stimulating hormone) in women of fertile age by clinical findings (amenorrhea or oligomenorrhea) associated with low 17-beta estradiol levels and low or normal gonadotropins and in postmenopausal women by the absence of high follicle-stimulating hormone levels. Growth hormone secretion was assessed using the following stimulation tests: insulin tolerance test or growth hormone-releasing hormone-arginine test, considering for the insulin tolerance test a cutoff value of $5.1 \mu \mathrm{g} / \mathrm{L}$ and for the growth hormone-releasing hormone-arginine test $4.1 \mu \mathrm{g} / \mathrm{L}$ [15]. Finally, diabetes insipidus was defined as emission of a high volume ( $>40 \mathrm{~mL} / \mathrm{kg} /$ day) of hypoosmotic urine.

\section{Statistical Analysis}

Categorical data are presented as percentage and proportion and were analyzed using the $\chi^{2}$ test or the Fisher exact test if the expected value was $<5$. Continuous parameters with normal distribution were described as mean \pm standard deviation and compared using the $t$ test. Non-Gaussian data were analyzed using median and interquartile range (IQR). Recovery of adrenal function 




Fig. 1. Cumulative probability of recovery of adrenal function in the two subgroups. AI, adrenal insufficiency.

and disease relapse were analyzed using survival analysis (KaplanMeier curves, log-rank test, and Cox regression, as appropriate). All statistical analyses were performed using SPSS 21 (IBM, Cary, NC, USA).

\section{Results}

Of 115 patients diagnosed with $\mathrm{CD}$ at our center and operated and followed between 1990 and 2017, 75 had AI at 2 months, the remaining 40 having one of the following conditions: normal pituitary function (10 patients), persistent $C D$ ( 16 patients), 2 were $<18$ years, and 6 had incomplete data. Of the 75 patients only 61 were followed for at least 3 years. The patients were divided into two groups according to the presence or absence of $\mathrm{CD}$ recurrence during follow-up. The patient characteristics are summarized in Table 1. In patients with negative imaging the confirmation of $\mathrm{CD}$ diagnosis was performed in 8 of 11 cases using inferior petrosal sinus sampling. In the remaining cases, the concordance between a positive corticotropin-releasing hormone test and a positive DXM 8 mg overnight suppression test was considered sufficient to justify the diagnosis of $\mathrm{CD}$. Of note,

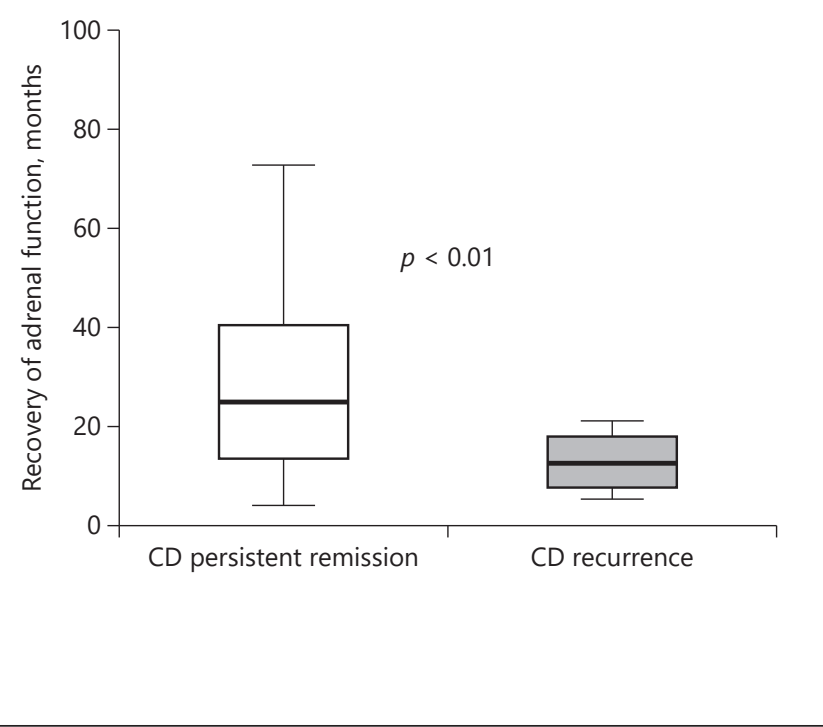

Fig. 2. Duration of AI in the two subgroups. AI, adrenal insufficiency; CD, Cushing disease.

4 patients had received previous therapies: 3 had a previous pituitary surgical intervention and treatment with ketoconazole, while 1 received only ketoconazole. Ketoconazole was discontinued the week before the surgical intervention. No patient had cranial or pituitary radiotherapy or pituitary radiosurgery before the surgical intervention or during follow-up, in the absence of CD recurrence. Of 3 patients who were reoperated only 1 had a total hypophysectomy.

The median follow-up was 6 years, ranging from 3 to 27 years (IQR 4-11). Overall, the median recovery time of adrenal function was 19 months (IQR 6-31), and it was the only variable that significantly differed between the two groups (Table 1). Of 61 patients with AI, $49 \%$ presented at least one additional pituitary deficiency. Growth hormone secretion was most frequently impaired (29.5\% of cases), followed by central hypothyroidism and hypogonadism with a similar distribution (21\% of cases) and lastly diabetes insipidus (Table 1). Also, 21.8\% (12/55) patients presented other TSS complications: 9 had intraoperative or postoperative cerebrospinal fluid leak, 1 hematoma, and 2 hyponatremia, with no difference between the two groups (Table 1). 
Table 2. Sensitivity, specificity, PPV, and NPV for the recovery time of AI predicting CD recurrence

\begin{tabular}{lllll}
\hline Recovery within 22 month & CD recurrence & Persistent remission & Total population & \\
\hline Yes & 10 & 10 & 20 & PPV 10/20 $=50 \%$ \\
No & 0 & 41 & 41 & NPV 41/41 $=100 \%$ \\
\hline & sensitivity $10 / 10=100 \%$ & specificity $41 / 51=80.4 \%$ & 61 & \\
\hline
\end{tabular}

AI, adrenal insufficiency; CD, Cushing disease; NPV, negative predictive value; PPV, positive predictive value.

\section{Recovery of Adrenal Function in Patients with Persistent Remission}

Fifty-one patients were still in remission at the last follow-up visit. As shown in Figure 1, the recovery rate of adrenal function in the persistent remission group was $37.3 \%(19 / 51)$ at 3 years and $55.8 \%(24 / 43)$ at 5 years, with a cumulative rate of $52.9 \%$ (27/51). At least one additional pituitary deficiency was detected in $52.9 \%$ (27/51) patients (Table 1). The recovery rate of adrenal function in patients with an otherwise normal postoperative pituitary function was $58.3 \%(14 / 24)$ at 3 years and $84.2 \%(16 / 19)$ at 5 years, with a cumulative rate of $70.8 \%(17 / 24)$.

Using univariate Cox regression, the recovery of adrenal function was negatively associated with younger age (OR 0.96, CI $0.92-0.99, p=0.015$ ) and absence of other pituitary deficits (OR 0.28 , CI $0.12-0.67, p=0.004$ ). In multivariate Cox regression that included age, hypopituitarism, duration of $\mathrm{CD}$ before surgical intervention, relative UFC cortisol post DXM 1 mg overnight prior to surgical intervention, and the year of TSS, the only significant predictor was residual pituitary function.

In patients who recovered adrenal function during follow-up, the median duration of AI was 25 months (IQR 13-40.9) and remained almost unchanged (25 months, IQR 12.6-47) after excluding 3 patients who had received previous therapies (ketoconazole, TSS). The duration of AI was positively correlated with the duration of CD before surgical intervention (Spearman correlation coefficient $0.42, p=0.014$ ), but it was not associated with the severity of cortisol hypersecretion (serum cortisol post DXM, relative UFC).

\section{Recovery of Adrenal Function in Patients with CD \\ Recurrence}

A total of 10 patients presented a recurrence during follow-up (16.4\%). The recurrence rate was 9.8\% (6/61) at 3 years and $16.3 \%(7 / 43)$ at 5 years, and the cumulative recurrence rate was $16.4 \%$ (10/61). Recurrence occurred

Postsurgical Recovery of Adrenal

Function in Cushing Disease between 0.6 and 16 years with a median of 1.2 years. The time needed to achieve recovery of adrenal function was significantly lower in the recurrence group compared to the persistent remission group (median AI time 12 vs. 25 months, $p=0.01$ ) (Table 1; Fig. 2). Cox regression at 10 years showed that the time needed to achieve recovery of adrenal function was negatively associated with disease recurrence (OR 0.91, CI 0.83-1, $p=0.05$ ). All patients with $\mathrm{CD}$ recurrence recovered their adrenal function within 22 months from surgery; recovery within 22 months had a positive predictive value (PPV) of $50 \%$ and a negative predictive value (NPV) of $100 \%$ (Table 2). The interval between recovery of adrenal function and CD recurrence varied from 0 to 15.5 years, with a median of 1.1 years.

\section{Discussion}

In this study we investigated the relationship between the time of postsurgical recovery of adrenal function and the risk of disease recurrence in a cohort of patients with $\mathrm{CD}$ treated at our center and followed for a minimum of 3 years. The cumulative recurrence rate in our study was $16.3 \%$. Recently, Fleseriu et al. [5] performed a review of 74 studies showing that recurrence rates ranged from 0 to $65 \%$ with a median of $10.6 \%$. The wide range was likely a consequence of high variability of the patient population included in each study, definition of recurrence, and duration of follow-up. Analyzing only those studies with similar criteria of recurrence to those used in the present study (high UFC, lack of suppression of low-dose DXM suppression test), a cumulative recurrence rate ranging from 9 to 34\% was reported [16-20]. In our group of patients, the recurrence rate increased with the length of follow-up, confirming that patients with CD in apparent remission after surgery need a potential lifelong followup. Notably, recurrence may occur even 16 years after the 
operation, although the median recurrence in our patients was after 1.2 years. In this particular case, we observed disease recurrence 15.5 years after normalization of adrenal function. Although in this patient the recovery of cortisol secretion might have had a physiological mechanism, for the moment it is difficult to define a period of time between the recovery of adrenal function and disease recurrence that may predict a physiological or a pathological recovery of the HPA axis.

Similarly, the recovery rate of adrenal function in patients with persistent disease remission increases during follow-up both in patients with and without other pituitary deficiencies, underlining the need for periodic reevaluation in order to avoid unnecessary chronic glucocorticoid replacement therapy. The median recovery time of AI in all patients was 19 months, similar to the mean recovery time reported in other studies that ranged between 11.4 and 25 months [8-11]. Of note, the studies mentioned above included also those cases that recurred during follow-up, but in these cases cortisol secretion has a tumoral origin, the recurrence being caused by the growth of a residual microscopic tumor [21]. Considering only those patients with persistent remission of $\mathrm{CD}$, the median recovery time of adrenal function was 25 months and was significantly longer in respect to patients who experienced disease recurrence. Another factor that could affect the duration of $\mathrm{AI}$ is previous therapy, in this case previous TSS and ketoconazole, therefore we reassessed the duration of AI, excluding the 4 pretreated patients, and the result was similar.

The recovery of adrenal function in patients persistently remitted during follow-up was associated with younger age at surgical intervention, in agreement with other studies $[7,8,22]$, while there is only one paper showing the contrary [11]. The presence of other pituitary insufficiencies, which represent one of the most common complications of pituitary surgery, had also a negative impact on the recovery of adrenal function, probably as a result of more aggressive and radical surgical intervention, as previously suggested [16]. In addition, as the quality of surgical intervention might have improved in the last decade as the neurosurgical technique changed and the experience of the surgical team increased, we investigated the impact of year when TSS was performed on the recovery of adrenal function, but no significant association was found.

Flitsch et al. [22] examined the preoperative findings of 86 patients with $\mathrm{CD}$ with different duration of hypocortisolism after surgery, showing that the recovery time of adrenal function during follow-up was positively cor- related with disease duration prior to surgical intervention, although this correlation did not reach statistical significance. Furthermore, in patients necessitating pharmacologic glucocorticoid treatment, the duration and cumulative dose of steroids were independent predictors of AI [23]. In our patients, the duration of AI was positively correlated with the duration of $\mathrm{CD}$, but not with the severity of cortisol hypersecretion. However, we were unable to retrospectively calculate the cumulative exposition to excess cortisol, which would probably be the most important parameter but is very difficult to calculate, requiring to take into account the severity for each year of exposition from the beginning of the disease (and not only from the time of diagnosis).

Most previous studies focused on the identification of predictive factors of remission or recurrence in patients with $\mathrm{CD}$. Even if these studies observed that recurrence was associated with the presence of macroadenoma [17], preoperative negative imaging of the tumor [24], younger age, and severity of cortisol secretion prior to surgical intervention $[25,26]$, in the present study the only predictive factor for $\mathrm{CD}$ recurrence was the duration of postsurgical AI. This finding is consistent with a recent report by Alexandraki et al. [8], who performed a single-center retrospective analysis of a large cohort of CD patients with a prolonged follow-up. All 10 patients who recurred recovered their adrenal function within 22 months, but in the meantime the same number of patients restored their adrenal function despite maintaining remission during follow-up. This observation confirms the results by Alexandraki et al. [8], who showed that recovery within 2 years had an NPV of $98.1 \%$ and a PPV of $59.3 \%$, with a slightly higher PPV probably as a consequence of a longer follow-up. In a similar way, Berr et al. [7] showed that in 54 patients with $\mathrm{CD}$, the recovery of adrenal function occurred within 3 years in case of subsequent disease recurrence.

The strengths of this study are the strict criteria of patient selection and the analysis of adrenal function recovery excluding patients with recurrent $\mathrm{CD}$. There are also some limitations to consider, in particular the retrospective character of the analysis, the relatively short minimum follow-up of 3 years, and the lack of data concerning late-night salivary cortisol, which were collected only for more recent patients and which could represent an early marker of the tumoral secretion of cortisol [27].

In conclusion, our study shows that the duration of AI after pituitary surgery in patients with $\mathrm{CD}$ is significantly shorter in recurrent $\mathrm{CD}$ than in the persistent remission group. After the exclusion of relapsed cases, the duration of 
AI assessed in the present study was slightly longer compared with previous studies and was negatively associated with younger age and absence of other pituitary deficiencies. Finally, the duration of AI may be a useful predictor of CD recurrence, and those patients who show a normal pituitaryadrenal axis within 2 years after surgery should be strictly monitored as they are at higher risk of disease relapse.

\section{Acknowledgment}

We thank Roberta Carminati and Arianna Cremaschi, medical students, for their aid in data collection.

\section{Statement of Ethics}

Subjects gave their written informed consent for the use of their clinical data for research purposes. Ethical committee approval was not required as patients were followed according to good clinical practice with no additional diagnostic or therapeutic interventions.

\section{Disclosure Statement}

The authors have nothing to disclose.

\section{Funding Sources}

This study was supported by the Associazione Italiana Ricerca sul Cancro (grant IG 2017-20594 to G. Mantovani) and the Ricerca Corrente Funds of the Italian Ministry of Health, Progetti di Ricerca di Interesse Nazionale (grant 2015ZHKFTA to G. Mantovani).

\section{Author Contributions}

A.L. Serban designed the study, analyzed the data, and wrote the manuscript. E. Sala, G. Carosi, and G. Del Sindaco collected and interpreted the data. G. Carosi and M. Locatelli analyzed the data and provided intellectual input. G. Mantovani, M. Arosio, C. Giavoli, and E. Ferrante devised the project and critically reviewed the manuscript. All authors approved the final version of the manuscript.

\section{References}

1 Plotz CM, Knowlton AI, Ragan C. The natural history of Cushing's syndrome. Am J Med. 1952 Nov; 13(5):597-614.

2 Clayton RN, Jones PW, Reulen RC, Stewart PM, Hassan-Smith ZK, Ntali G, et al. Mortality in patients with Cushing's disease more than 10 years after remission: a multicentre, multinational, retrospective cohort study. Lancet Diabetes Endocrinol. 2016 Jul;4(7):569-76.

3 Petersenn S, Beckers A, Ferone D, van der Lely A, Bollerslev J, Boscaro M, et al. Therapy of endocrine disease: outcomes in patients with Cushing's disease undergoing transsphenoidal surgery: systematic review assessing criteria used to define remission and recurrence. Eur J Endocrinol. 2015 Jun; 172(6):R227-39.

4 Jagannathan J, Smith R, DeVroom HL, Vortmeyer AO, Stratakis CA, Nieman LK, et al. Outcome of using the histological pseudocapsule as a surgical capsule in Cushing disease. J Neurosurg. 2009 Sep;111(3):531-9.

5 Fleseriu M, Hamrahian AH, Hoffman AR, Kelly DF, Katznelson L; AACE Neuroendocrine and Pituitary Scientific Committee. American Association of Clinical Endocrinologists and American College of Endocrinology disease state clinical review: diagnosis of recurrence in Cushing disease. Endocr Pract. 2016 Dec;22(12):1436-48.
6 van Haalen FM, Broersen LH, Jorgensen JO, Pereira AM, Dekkers OM. Management of endocrine disease: Mortality remains increased in Cushing's disease despite biochemical remission: a systematic review and metaanalysis. Eur J Endocrinol. 2015 Apr; 172(4):R143-9.

7 Berr CM, Di Dalmazi G, Osswald A, Ritzel K, Bidlingmaier M, Geyer LL, et al. Time to recovery of adrenal function after curative surgery for Cushing's syndrome depends on etiology. J Clin Endocrinol Metab. 2015 Apr; 100(4):1300-8.

8 Alexandraki KI, Kaltsas GA, Isidori AM, Storr HL, Afshar F, Sabin I, et al. Long-term remission and recurrence rates in Cushing's disease: predictive factors in a single-centre study. Eur J Endocrinol. 2013 Mar;168(4): 639-48.

9 Costenaro F, Rodrigues TC, Rollin GA, Ferreira NP, Czepielewski MA. Evaluation of Cushing's disease remission after transsphenoidal surgery based on early serum cortisol dynamics. Clin Endocrinol (Oxf). 2014 Mar; 80(3):411-8.

10 Aranda G, Enseñat J, Mora M, Puig-Domingo M, Martínez de Osaba MJ, Casals G, et al. Long-term remission and recurrence rate in a cohort of Cushing's disease: the need for longterm follow-up. Pituitary. 2015 Feb;18(1): 142-9.
11 Prete A, Paragliola RM, Bottiglieri F, Rota CA, Pontecorvi A, Salvatori R, et al. Factors predicting the duration of adrenal insufficiency in patients successfully treated for Cushing disease and nonmalignant primary adrenal Cushing syndrome. Endocrine. 2017 Mar; 55(3):969-80.

12 Levi V, Bertani GA, Guastella C, Pignataro L, Zavanone ML, Rampini PM, et al. Microscopic versus endoscopic transsphenoidal surgery for pituitary adenoma: analysis of surgical safety in 221 consecutive patients. Clin Otolaryngol. 2017 Apr;42(2):466-9.

13 Almutairi RD, Muskens IS, Cote DJ, Dijkman MD, Kavouridis VK, Crocker E, et al. Gross total resection of pituitary adenomas after endoscopic vs. microscopic transsphenoidal surgery: a meta-analysis. Acta Neurochir (Wien). 2018 May;160(5):1005-21.

14 Ambrosi B, Barbetta L, Re T, Passini E, Faglia $\mathrm{G}$. The one microgram adrenocorticotropin test in the assessment of hypothalamic-pituitary-adrenal function. Eur J Endocrinol. 1998 Dec;139(6):575-9.

15 Molitch ME, Clemmons DR, Malozowski S, Merriam GR, Vance ML; Endocrine Society. Evaluation and treatment of adult growth hormone deficiency: an Endocrine Society clinical practice guideline. J Clin Endocrinol Metab. 2011 Jun;96(6):1587-609. 
16 Alwani RA, de Herder WW, van Aken MO, van den Berge JH, Delwel EJ, Dallenga AH, et al. Biochemical predictors of outcome of pituitary surgery for Cushing's disease. Neuroendocrinology. 2010;91(2):169-78.

17 Dimopoulou C, Schopohl J, Rachinger W, Buchfelder M, Honegger J, Reincke M, et al. Long-term remission and recurrence rates after first and second transsphenoidal surgery for Cushing's disease: care reality in the $\mathrm{Mu}$ nich Metropolitan Region. Eur J Endocrinol. 2013 Dec;170(2):283-92.

18 Hassan-Smith ZK, Sherlock M, Reulen RC, Arlt W, Ayuk J, Toogood AA, et al. Outcome of Cushing's disease following transsphenoidal surgery in a single center over 20 years. J Clin Endocrinol Metab. 2012 Apr;97(4): 1194-201.

19 Pereira AM, van Aken MO, van Dulken $H$, Schutte PJ, Biermasz NR, Smit JW, et al. Long-term predictive value of postsurgical cortisol concentrations for cure and risk of recurrence in Cushing's disease. J Clin Endocrinol Metab. 2003 Dec;88(12):5858-64.
20 Jehle S, Walsh JE, Freda PU, Post KD. Selective use of bilateral inferior petrosal sinus sampling in patients with adrenocorticotropin-dependent Cushing's syndrome prior to transsphenoidal surgery. J Clin Endocrinol Metab. 2008 Dec;93(12):4624-32.

21 Lonser RR, Nieman L, Oldfield EH. Cushing's disease: pathobiology, diagnosis, and management. J Neurosurg. 2017 Feb;126(2): 404-17.

22 Flitsch J, Lüdecke DK, Knappe UJ, Saeger W. Correlates of long-term hypocortisolism after transsphenoidal microsurgery for Cushing's disease. Exp Clin Endocrinol Diabetes. 1999; 107(3):183-9.

23 Sacre K, Dehoux M, Chauveheid MP, Chauchard M, Lidove O, Roussel R, et al. Pituitaryadrenal function after prolonged glucocorticoid therapy for systemic inflammatory disorders: an observational study. J Clin Endocrinol Metab. 2013 Aug;98(8):3199-205.
24 Keskin FE, Ozkaya HM, Bolayirli M, Erden S, Kadioglu P, Tanriover N, et al. Outcomes of Primary Transsphenoidal Surgery in Cushing Disease: Experience of a Tertiary Center. World Neurosurg. 2017 Oct;106: 374-81.

25 Sonino N, Zielezny M, Fava GA, Fallo F, Boscaro M. Risk factors and long-term outcome in pituitary-dependent Cushing's disease. J Clin Endocrinol Metab. 1996 Jul;81(7):2647-52.

26 Shirvani M, Motiei-Langroudi R, Sadeghian H. Outcome of Microscopic Transsphenoidal Surgery in Cushing Disease: A Case Series of 96 Patients. World Neurosurg. 2016 Mar;87: $170-5$.

27 Danet-Lamasou M, Asselineau J, Perez P, Vivot A, Nunes ML, Loiseau $\mathrm{H}$, et al. Accuracy of repeated measurements of late-night salivary cortisol to screen for early-stage recurrence of Cushing's disease following pituitary surgery. Clin Endocrinol (Oxf). 2015 Feb; 82(2):260-6. 